\title{
POLÍTICAS Y CONFLICTOS SOCIO AMBIENTALES: EL CASO DE LA TENENCIA DE LA TIERRA Y LOS MONOCULTIVOS EN EL CARIBE DE COSTA RICA (2006-2012)
}

\section{SOCIO ENVIRONMENTAL POLICIES AND CONFLICTS: THE CASE OF LAND TENURE AND MONOCULTURES IN COSTA RICAN CARIBBEAN (2006-2012)}

\author{
José Julián Llaguno* \\ Sindy Mora Solano** \\ Ana Lucía Gutiérrez Espeleta** \\ Priscilla Barrios Alfaro \\ Flavio Mora Moraga*
}

RESUMEN

\begin{abstract}
El artículo tiene como objetivo caracterizar los principales conflictos socios ambientales del Caribe costarricense en el periodo 2006-2012. Este proceso se va a presentar a partir de una tensión principal, entre el Estado y los pobladores locales, quienes mantienen varias disputas alrededor de una serie de políticas que modifican las formas de tenencia de la tierra, así como, la falta de regulaciones en los sistemas de monocultivo de la piña y el banano.
\end{abstract}

\footnotetext{
PALABRAS CLAVE: COSTA RICA * MEDIO AMBIENTE * CULTIVO * PIÑA * BANANOS * ACCIONES COLECTIVAS
}

\footnotetext{
* Instituto de Investigaciones Sociales (IIS) de la Universidad de Costa Rica (UCR). jllaguno@gmail.com

** Instituto de Investigaciones Sociales (IIS) de la Universidad de Costa Rica (UCR). sindymora@gmail.com

*** Instituto de Investigaciones Sociales (IIS) de la Universidad de Costa Rica (UCR). ana.gutierrez@ucr.ac.cr

***** Escuela de Antropología de la Universidad de Costa Rica (UCR), estudiante. priscilunaytica@gmail.com

****** Maestría Centroamericana en Historia de la Universidad de Costa Rica (UCR), estudiante. jf2m013@gmail.com
} 


\section{ABSTRACT}

The article makes a characterization of the main socio-environmental conflicts in the Costa Rican Caribbean in the period 2006-2012. This process will be presented from a main conflict between the state and local villagers, who maintain several disputes about a series of policies that modify the forms of land tenure and the lack of regulations in systems of pineapple and banana monoculture.

KEYWORDS: COSTA RICA * ENVIRONMENT * CONFLICT * CULTIVATION * PINEAPPLES * BANANA TREES * COLLECTIVE ACTIONS

\section{INTRODUCCIÓN}

Este artículo tiene como objetivo el hacer una caracterización de los principales conflictos socio ambientales en el Caribe costarricense en los dos últimos gobiernos (20062012). El papel del Estado es uno de los principales actores que potencia y profundiza estos conflictos, a partir del desarrollo de un conjunto de medidas que facilitan la inseguridad en la tenencia de la tierra, así como, la falta de regulaciones en los sistemas de monocultivo de piña $y$ banano.

Esta relación se va a fundamentar en la revisión, síntesis y crítica de cuatro tipos de fuentes: artículos de prensa ${ }^{1}$, testimonios ${ }^{2}$, legislación ${ }^{3}$, así como artículos y libros académicos. Estos elementos se articulan en tres ejes de discusión principal: la situación de la tenencia de la tierra, el estado del monocultivo de banano $y$ piña, $y$ las formas de resistencia comunitaria y laboral frente a estos conflictos. La última parte busca ubicar estos elementos en el contexto actual para delimitar en qué medida el sistema de extracción de recursos y desarrollo de la lógica de plantación extensiva,

1 Como principal referente se consultó el periódico Semanario Universidad.

Se toman en cuenta los criterios de varias personas activistas $y$ dirigentes vinculados a diferentes organizaciones del Caribe.

Se refiere a proyectos y leyes aprobadas en el periodo 2006-2012, que afectan la zona de estudio. se mantiene como constante en el Caribe costarricense (Bourgois, 1994).

La iniciativa de articular estos elementos surge de la discusión y el intercambio de tres proyectos específicos ${ }^{4}$ que han trabajo en distintas comunidades del Caribe con temáticas como: memoria colectiva, acciones colectivas, agroecología y lucha por la tierra. Esta experiencia práctica hace necesaria una discusión más amplia sobre la construcción de la región en función de su papel geopolítico y cultural. Esto se traduce en un mantenimiento y profundización de conflictos socio ambientales que se articulan a una lógica de construcción económica del Caribe basado en la agroindustria de exportación.

Esta situación se ha profundizado en el caso de la extensión del monocultivo de piña en los últimos años, por lo que esta propuesta busca ver las diferentes formas de intervención del Estado en estos conflictos. Con esta perspectiva, se busca transcender la lógica parcelaria de la investigación en proyectos específi$\cos y$ articular una propuesta de interrogación sobre algunos temas clave que atraviesan al Caribe en la actualidad.

Estos son dos proyectos de acción social que trabajan en los cantones de Guácimo y Sixaola, y uno de investigación que articula una perspectiva del Caribe en su conjunto sobre ex trabajadores bananeros. Todos estos se encuentran inscritos en el programa Nuevas Formas de Acumulación, Distribución y Desigualdad (PADD) del Instituto de Investigaciones Sociales de la Universidad de Costa Rica. 
Esta reflexión se encuentra enmarcada en un contexto nacional de profundización de las formas de neo liberalización de la naturaleza, a partir de la puesta en vigencia del Tratado de Libre Comercio con Estados Unidos en el 2008. En este se articulan algunos de los elementos principales de la estrategia de mercantilización que incluye: bioprospección, semillas modificadas, extensión de derechos de propiedad intelectual, aumento de la importación de alimentos y fomento de agro exportación. Todas estas medidas favorecen los cambios institucionales y jurídicos que buscan desarrollar las condiciones necesarias para ampliar los circuitos de acumulación de capital (Rodríguez, S., 2012).

Este panorama general, se traduce en políticas y medidas específicas para la región Caribe que articula a este espacio con el mercado mundial. Es importante mencionar que esta articulación entre la región y los circuitos de acumulación capitalista mundiales viene desde finales del siglo xix con el desarrollo de la lógica de la plantación del banano. Desde esa época, la provincia de Limón ha tenido un papel estratégico para el Estado, puesto que a través de esta, es donde circulan la mayoría de mercancías de exportación e importación del país (Montero y Viales, 2011).

Además de este punto, la región concentra una importante cantidad de recursos naturales $y$ en biodiversidad que han sido explotados por diferentes instituciones estatales $y$ empresas transnacionales. Estos dos elementos han coadyuvado a que el Caribe se construya como uno de los principales circuitos de circulación de capital. Esta situación la demuestra la cantidad de obras de infraestructura e inversión que se han propuesto en los últimos gobiernos como: la concesión de los muelles públicos, la ampliación de una refinería de petróleo con capital chino, los proyectos de desarrollo de marinas de atraque turístico y la construcción de torres y líneas de alta tensión (Ruiz, 2013).

Esta infraestructura tiene la función doble de servir como instrumento para la extracción de recursos estratégicos como minerales, petróleo y agua, así como facilitar la circulación de bienes energéticos para alimentar otros proyectos de mayor envergadura como el plan Mesoamérica o la ampliación del Canal de Panamá. Estos proyectos no se han desarrollado de forma monolítica $y$ han encontrado modificaciones $y$ resistencia de parte de actores locales y regionales. Parte de los objetivos de este texto es mostrar algunas de estas acciones y cómo han incidido en distintos niveles, en el curso de estas modificaciones actuales.

\section{SITUACIÓN DE LA TENENCIA DE LA TIERRA EN EL CARIBE 2006-2012}

El siguiente apartado tiene el objetivo de hacer una síntesis sobre las principales problemáticas ligadas a la tenencia de la tierra en el Caribe costarricense. Se van a caracterizar los conflictos principales a partir de una reflexión entre las $y$ los actores involucrados y sus perspectivas $y$ diferencias. Para lograr esto se va a partir de una revisión de la prensa, la legislación y los documentos de organizaciones de base de la zona.

Los principales conflictos se localizan en el área geográfica denominada Caribe Sur, que es la zona que incluye diferentes tipos de regulación del espacio como: zona marítimoterrestre, territorios indígenas, asentamientos campesinos, zonas protegidas y frontera. Cada uno de estos tiene un tipo de regulación diferente e involucra a una serie de actores como: instituciones estatales, organizaciones no gubernamentales, empresas y grupos de pobladores locales (Rodríguez, S., 2012).

Cada uno de estos sectores tiene una forma específica de entender el uso y regulación del espacio, por lo que las diferencias actuales en su conformación tienen que ver con una interacción entre problemas históricos y cambios recientes en la delimitación de este territorio. En términos de configuración administrativa, esa zona se encuentra delimitada por el cantón de Talamanca, el cual tiene una extensión de $2810 \mathrm{~km}^{2}$ y se divide en los distritos de Bratsi, Sixaola, Cahuita y Telire (Borge, 1998).

El espacio dedicado a la protección ambiental constituye el 55\% del territorio, en diferentes modalidades de protección como: parques, reservas y refugios. Los más importantes son el Parque Nacional Cahuita, Parque Nacional La Amistad y el Refugio de Vida 
Silvestre Gandoca-Manzanillo (Municipalidad de Talamanca, 2003). La institución encargada de regular estas zonas es el Ministerio de Ambiente, Energía y Telecomunicaciones (міNAET) a través del Sistema Nacional de Áreas de Conservación (sinac).

Dentro de este sistema de regulación, han surgido una serie de conflictos en los últimos años, ligados a la forma de delimitación $y$ administración de las zonas protegidas, principalmente, sobre el Refugio Gandoca-Manzanillo. Esta situación detona con la orden de demolición en el 2011, de los hoteles Las Palmas y Suerre, propiedad del empresario checo Jan Kalina. Este fue la culminación de un largo proceso que venía llevando el Tribunal Ambiental y el sinac, por el cambio del uso, la invasión de la zona marítima terrestre y el daño ambiental ocasionado al refugio por parte del hotel (Ramírez, E., 13/07/2011).

Esta acción ha producido una serie de desencuentros entre las instituciones gubernamentales, grupos ambientalistas, pobladores locales y empresarios. Parte de estas diferencias se relacionan con el precedente jurídico que establece la demolición de los hoteles, lo cual pone en una situación de incertidumbre a otros pobladores locales que viven cerca del refugio. Asimismo, denota los intereses económicos de una serie de empresarios con proyectos de fuerte inversión, como el fallecido un mes después de la acción, Jan Kalina (Ramírez, E., 13/07/2011).

Esta diferenciación se ha traducido al ámbito jurídico a partir de la introducción de dos proyectos de ley, "Reconocimiento de los derechos de los habitantes del Caribe", expediente 18207 y "Declaratoria de Cahuita y Puerto Viejo como ciudad", expediente 18425, presentados en el 2011 y 2012, respectivamente. Estos han sido propuestos por el diputado Walter Céspedes, representante de la provincia de Limón por el Partido Unidad Social Cristiana (pusc). El primer proyecto tiene por objetivo sacar de la zona del refugio las comunidades de: Gandoca, Mata de Limón, San Miguel, Manzanillo y Puerto Viejo, así como permitir la titulación de tierras y el desarrollo de actividades socioeconómicas en la zona marítimo-terrestre (Céspedes, 2011).
Este proyecto no deja claro la magnitud de la actividad que se pretende legitimar $y$ desarrollar en el lugar y se relaciona con otro texto presentado por el mismo diputado, el cual busca declarar las comunidades de Puerto Viejo y Cahuita como ciudades. Este cambio permitiría que la Municipalidad de Talamanca otorgue concesiones por 99 años para: construcción de muelles o bien, otras instalaciones industriales, comerciales, institucionales o habitacionales en los terrenos a que se refiere la presente ley. Así, como concesiones de carácter: turístico, cultural, deportivo, recreativo o para el desarrollo de actividades productivas (Céspedes, 2012).

De aprobarse estos proyectos, el sistema de regulación del espacio de estas comunidades cambiaría hacia uno que permitiría un crecimiento mayor de las actividades económicas extensivas en la costa, ya que en ninguno de los proyectos se delimita explícitamente el tamaño y carácter de las explotaciones económicas. Esta situación mantiene en pugna a diferentes sectores ambientalistas, de pobladores locales $y$ empresarios que desarrollan distintas interpretaciones sobre el uso del espacio (Ramírez, E., 13/07/2011).

Un segundo conflicto está asociado a la delimitación de los territorios indígenas, estos cubren el 30\% del cantón. Los más importantes son Bratsi, Telire y Keköldi (Municipalidad de Talamanca, 2003) en donde conviven los pueblos Bribri y Cabecar. Según datos del Ministerio de Agricultura y Ganadería (мас) (2007: 27), la posesión efectiva del territorio en manos indígenas es: $34 \%$ para la población cabecar, $75 \%$ para los Bribris que viven en Bratsi y Telire, $y$ $50 \%$ para los bribris que habitan Keköldi. Estos datos demuestran la heterogeneidad interna de situaciones que en general se ven afectadas por: la posesión de tierras de no indígenas, la restricción de áreas protegidas y la falta de ordenamiento jurídico de muchas de estas tierras ${ }^{5}$.

\footnotetext{
5 A pesar de esta situación, Talamanca es el que presenta el mayor porcentaje de posesión indígena de la tierra de los 24 territorios establecidos en el país desde los años 70 .
} 
El manejo de estos territorios es función de las Asociaciones de Desarrollo Indígena (ADI) creadas mediante la ley indígena de 1977, que establecía a estas como las instancias de gobierno local indígena, sin tomar en cuenta las formas de organización y cosmovisión local. La regulación de estos territorios estaba en manos del Instituto de Desarrollo Agrario (IDA), institución que desarrolla un programa de traspaso que empieza en 1975. Para el 2008, el 73\% de las tierras estaban inscritas a nombre de las diferentes asociaciones (Mora-Alfaro, 2006: 85).

Este traspaso de competencias y potestades institucionales no ha estado exento de conflictos. Uno de los casos actuales que más permanece es el del territorio Keköldi, cercano a la costa, el cual ha pasado por una serie de ampliaciones $y$ restricciones desde los años 70 y llevó a parte de sus pobladores a enfrentarse con un empresario italiano que reclamaba 50 hectáreas, durante el 2011 (Chacón, 10/08/2011). La permanencia de este conflicto ha generado disputas entre los Bribris con empresarios extranjeros y pobladores locales, como los negros de la costa y campesinos de la comunidad de Margarita en Sixaola (Córdoba, 14/04/2011). El uso del espacio, la relación directa con los recursos naturales y las prácticas culturales, son algunos elementos que inciden en estas tensiones entre los pobladores.

El tercer conflicto principal, tiene que ver con la titulación de tierras dentro de los 19 asentamientos campesinos ubicados en el distrito de Sixaola, colindante con la frontera con Panamá. Estos fueron creados a partir de los años 70, producto de la migración de campesinos y campesinas sin tierra, obreros proletarizados $y$ ex trabajadores bananeros de Nicaragua, Guanacaste y otras zonas del Caribe (Fernández, 1989). El IDA fue la institución encargada de crear los asentamientos y ordenar el territorio. Según los datos presentados en el 2005 , el $84 \%$ de estos lugares fueron producto de ocupación precaria y el $89 \%$ de las parcelas tienen algún nivel de titulación (Mora, 2006).

Desde el 2010, parte de los pobladores de Sixaola han mantenido un conflicto importante con la institución, debido a que varios pronunciamientos de la Contraloría de la República obligan al IDA a anular el programa de titulación y brindar arrendamientos. Esta política se generaliza a partir de la aprobación de la Ley 9036, la cual crea el Instituto de Desarrollo Rural (INDER), cuya finalidad es otorgar arrendamientos a todos los beneficiarios del programa de asentamientos de la institución (Llaguno y Picado, 2011).

Este cambio sumado a la legislación fronteriza pone en una situación de vulnerabilidad permanente a este sector que suma alrededor de 15000 personas, la mitad de la población del cantón (Chacón, 13/07/2011). Esto aunado al conflicto de delimitación del territorio Keköldi que colinda con varios de estos asentamientos, mantiene a la población en una constante tensión por la tierra, debido a la falta de información clara sobre la legislación y sus cambios.

Tomando en cuenta esta problemática, existe en la Asamblea Legislativa, un proyecto de ley denominado "Concesión de la zona fronteriza con Panamá”, nro. 17 956, el cual tiene como objetivo crear una figura jurídica intermedia entre la titulación y el arrendamiento, con el fin de que los pobladores de Sixaola puedan tener una mayor seguridad jurídica. Este sistema permite una concesión de la $\mathrm{Mu}$ nicipalidad para personas que hayan venido ocupando la tierra por más de diez años (Asamblea Legislativa, proyecto de Ley nro. 17 956, 2010). Esta forma le daría una mayor potestad al municipio para la administración de estos territorios, el cual tendría que tener vigente un plan regulador.

Para que esto sea posible, el proyecto tiene que garantizar la reducción de la zona fronteriza con Panamá de 2000 a 200 metros. Esta franja de territorio constitucionalmente, pertenece al Estado y está delimitada para garantizar la seguridad nacional. Sobre esta propuesta, la Controlaría de la República ha desarrollado varios pronunciamientos en contra, así como, varios grupos campesinos que ven en esta alternativa una solución media a su problemática, ya que no reconoce el derecho a la titulación después de más de veinte años de ocupación de la tierra, en la mayoría de los casos (Chacón, 13/07/2011). Esta limitación legal, las diferencias de intereses entre los 
pobladores de los asentamientos y las instituciones estatales, sumado a la posición estratégica que significa para el Estado el control de las fronteras, mantiene un conflicto abierto que se mantiene hasta la actualidad.

Según la información presentada, la permanencia de estos conflictos, en el Caribe Sur, se debe a varios factores históricos y presentes. En el primer caso, se mantiene una constante disputa por el espacio desde la introducción de la lógica de plantación bananera en el siglo xix que desplazó a gran parte de la población nativa de los valles de Talamanca. El segundo momento de tensión importante se dio a partir de la delimitación administrativa del territorio en 1970, con la creación del cantón. Este trajo una serie de regulaciones del espacio que involucraban una gran cantidad de instituciones estatales diversas.

De esta manera, ha permanecido una diferenciación entre los actores locales y externos debido a las distintas concepciones, regulaciones e interacciones de los espacios que conforman el territorio. Esta situación, permanece en la actualidad, debido al cambio en la dinámica socioeconómica y estratégica de la región, la cual mantiene una gran cantidad de recursos importantes como: biodiversidad, minerales y atracciones naturales para el turismo.

Esta combinación de factores coadyuva a la permanencia de disputas por el territorio que favorecen la elaboración y aprobación de diferentes modalidades de legislación. Estas a su vez, son el resultado de un amplio movimiento de intereses que mantiene en tensión los espacios de regulación del territorio. Tomar en cuenta esta complejidad de factores es vital para desarrollar una reflexión regional en relación con una política ambiental nacional que ha tendido a favorecer los conflictos en los últimos años.

\section{SITUACIÓN DE LA PRODUCCIÓN BANANERA EN EL CARIBE 2006-2012}

En este apartado se analizan las principales características de la conflictividad originada por la producción y la expansión bananera en la región Caribe, para exponer, posteriormente, los rasgos que ha adquirido esta conflictividad a partir del año 2006, periodo que ocupa este trabajo.

Desde sus inicios, la producción bananera tuvo significativas consecuencias en el ambiente, en las condiciones laborales y en la salud de la población trabajadora. Así, la producción bananera no solo tuvo un enorme impacto ambiental en las regiones productoras, a través de la deforestación y el desarrollo de enfermedades como el mal de Panamá, la sigatoga $y$ los nematodos, sino que las características de la producción de este monocultivo se tradujeron en grandes riesgos que tuvieron sus efectos negativos en la salud de la población trabajadora (Giralt, 1996; Marquardt, 2001 y 2003).

En ese sentido, si bien, desde los inicios de la instalación de las bananeras se han vivido diversos ciclos de organización y movilización política por parte de estos trabajadores, en este trabajo se hará referencia a aquellos ciclos que se dieron a partir de la entrada en vigencia del Plan de Fomento Bananero, en 1985. A partir de este año, empezó a desarrollarse este Plan, que significó una exención de impuestos y regalías fiscales a las empresas bananeras, a fin de fomentar la expansión productiva del banano en la región. Esta expansión bananera implicó, en primer lugar, la disponibilidad de nuevas tierras, a través de la compra de estas y las presiones para la venta de las tierras propiedad de pequeños productores. En segundo lugar, implicó la disponibilidad de créditos bancarios para la consecución de las tierras y la compra de paquetes tecnológicos para la aplicación de plaguicidas. Finalmente, dicha expansión supuso la disponibilidad de mano de obra que se insertó en las bananeras, proceso que se vio acompañado de un significativo impulso al solidarismo, como mecanismo organizativo de las y los trabajadores bananeros de la región (Hermosilla, 1998).

De acuerdo a datos provenientes de la carta pastoral de Monseñor Alfonso Coto Alfaro (2003), la expansión bananera supuso un aumento de 8000 hectáreas cultivadas de banano en 1985 a 21000 hectáreas de banano en el año 1989. Así, datos para 1998 muestran que, de los 80000 trabajadores bananeros existentes en ese momento, únicamente 15000 de estos 
contaban con un empleo formal (Hermosilla, 1998) y solo para 1990 , el $78 \%$ de las intoxicaciones de trabajadores por el uso de agroquímicos en el país se encontraban localizadas en comunidades como Guácimo (intoxicaciones directamente vinculadas a la producción bananera) (Amador, 1998).

Estas condiciones laborales no eran radicalmente distintas para las mujeres trabajadoras de las bananeras. En un diagnóstico de la situación de la mujer trabajadora bananera, realizado por Quesada, la autora señala la persistencia de problemas que las mujeres enfrentaban y enfrentan todavía hoy: la pobreza, la baja remuneración y la diferenciación salarial respecto de los hombres, la ausencia de alternativas laborales en la región, la ausencia del derecho a la libre sindicalización, así como, el acoso y abuso sexual en las bananeras (Quesada, 1998).

De la misma manera, en el marco del Plan de Fomento Bananero prevalecía una fuerte represión de la organización política de los trabajadores, que iba desde la amenaza verbal hasta los despidos por simpatizar con la organización sindical, la continua elaboración de listas negras y la no contratación de trabajadores sindicalizados, las amenazas de muerte $y$ la agresión física (Coordinadora de Sindicatos Bananeros, 1998).

Fue en este escenario de condiciones y consecuencias de la producción bananera, que el 25 de diciembre de 1989, el Obispo de Limón, Monseñor Alfonso Coto, emitió una Carta Pastoral titulada "Alto a la Expansión Bananera Incontrolada", que posteriormente, en el año 1992, posibilitaría el surgimiento del Foro Emaús (Coto, 2003). Fue también en 1992 que se realizó una marcha conocida como la "Marcha por la Vida y los Derechos Humanos", inspirada en la Carta Pastoral de 1989. Como señala Hermosilla, estas iniciativas "contribuyeron a animar y consolidar la idea de que el Foro Emaús era un espacio viable y necesario para luchar por los intereses populares en el marco de amplitud, democracia y ecumenismo popular" (1998: 12).

Así, el Foro Emaús fue una propuesta organizativa que permitió plantear una fuerte discusión, con acciones concretas de denuncia a las violaciones laborales que se realizaron en la región en el ámbito de la producción bananera. Sin embargo, esta iniciativa permitió que se estableciera una red de organizaciones de carácter eclesiástico, comunal, ambiental, indígena y sindical, con la participación de organizaciones no gubernamentales, la cual culminó su accionar en el año 2009, fundamentalmente, por razones de financiamiento (Barraza, Jansen, van Wendel de Joode y Wesseling, 2013). A pesar de las problemáticas laborales, ambientales y en la salud de los habitantes de la región, muchos siguen lamentando la desaparición del Foro Emaús como forma organizativa, considerando que los problemas relacionados con la producción bananera no se han acabado y también, la producción piñera ha profundizado muchas de las problemáticas citadas (Entrevista a integrante y fundador del Foro Emaús, 23/01/2012).

Ahora bien, desde inicios de los años 90 hasta la actualidad, se ha mantenido un significativo movimiento de trabajadores bananeros afectados por el nemagón (Giralt, 1996 y Mora, 2013). Este movimiento se ha basado en la búsqueda de la satisfacción de la demanda de una indemnización para los trabajadores bananeros que se vieron afectados por este agroquímico, indemnización que ha sido fundamentalmente, asumida por el Estado costarricense. Sin embargo, a pesar de que se estima que en Costa Rica hubo 30000 afectados por el nemagón, diversas entrevistas realizadas muestran que no se constituyó un fuerte movimiento de trabajadores, dada la importancia que adquirió la indemnización económica en el marco de estas luchas, dejando de lado las reivindicaciones en torno a la salud de los trabajadores, de sus hijos, hijas o esposas; abandonando las afectaciones de carácter psicológico y dejando sin crítica alguna, la utilización de agroquímicos $y$ los efectos que estas sustancias tienen en el ambiente, incluso en el marco de nuevos monocultivos (Entrevista a integrante $y$ fundador del Foro Emaús, 23/01/2012 y entrevista a integrante fundadora del Foro Emaús, 11/07/2012).

A pesar del escenario descrito anteriormente, son pocas las acciones de protesta desarrolladas en la región en el tema de las bananeras $y$ en el periodo definido para este 
trabajo. Así, por ejemplo, las principales acciones de protesta fueron protagonizadas por los extrabajadores bananeros afectados por el nemagón, quienes protestaron durante el año 2009 , con el fin de solicitar la realización de exámenes médicos a los hombres para que se les comprobara la esterilidad. Estos extrabajadores también demandaron el nombramiento de una doctora encargada de realizar dicho trabajo, dado que el Instituto Nacional de Seguros (INs) ya no estaba realizando esa labor (Comité de Seguimiento del Conflicto Social, 2009).

Posteriormente, en el año 2010, estos extrabajadores interpusieron un recurso de amparo, ya que el ins había dejado de realizar los exámenes médicos a los afectados por el nemagón, desde meses anteriores. En este caso, la Sala iv falló positivamente al recurso interpuesto por los extrabajadores, considerando que a la fecha aún se encontraban pendientes los exámenes de unas 2000 personas. A partir del fallo, el ins debió empezar a realizar el pago a los extrabajadores (Díaz, 22/09/2010).

De la misma manera, el 11 de agosto de 2011, la empresa bananera dole firmó un arreglo extrajudicial con 780 extrabajadores bananeros afectados por el nemagón, del cual se desconoce el monto indemnizado para estos, lo cual supuso el cese de acciones de protesta o de carácter legal contra la empresa (Villegas, 12/08/2011).

Finalmente, una de las acciones de protesta destacables de este periodo refiere a la huelga protagonizada por la Asociación de Trabajadores Guaymíes de Sixaola y el Comité Permanente de Trabajadores. Esta huelga inició el 10 de noviembre de 2011 y finalizó el 30 de noviembre de 2011, en contra de la empresa Banana Development Corporation (BANDEco), subsidiaria de la empresa Del Monte. La principal demanda de esta huelga fue la negociación de un nuevo arreglo directo con la empresa. Después de varios días de huelga, la empresa anunció el establecimiento de reuniones con los representantes de los trabajadores para negociar el arreglo directo, comprometiéndose a no tomar represalias (Barquero, 10/11/2011 y 01/12/2011).
Ante las pocas acciones de protesta que se registraron en este periodo en el tema de las condiciones $y$ la producción bananera, y a pesar de que persisten las condiciones laborales que violan las disposiciones legales mínimas, se puede señalar, a manera de hipótesis, que en la región Caribe hay una especie de "naturalización" respecto a las condiciones laborales en las bananeras, las que son aceptadas como "malas"; es decir, persisten en la región las ideas de que la producción bananera genera abusos hacia la población trabajadora, costarricense o migrante, que genera discriminación hacia las mujeres, que supone bajas remuneraciones, accidentes laborales y contaminación, lo cual no se ha acompañado de una fuerte movilización social. A esto se une el hecho de que las fuentes de trabajo son escasas, por lo que aceptar los trabajos disponibles supone estas condiciones laborales, en las que existe una reducida posibilidad de articular iniciativas organizativas que permitan la reivindicación de las demandas. Adicionalmente, para complementar esta hipótesis, puede haber un significativo subregistro de acciones de protesta en los medios de comunicación, a lo que se une el hecho de que se le ha dado prioridad a la movilización social comunitaria vinculada a la producción piñera, lo cual ha convocado a distintas comunidades $y$ actores de la región.

A pesar de lo anterior, un entrevistado afectado por el nemagón y quien actualmente se mantiene trabajando en las bananeras, señaló que las condiciones laborales de estas persisten, de allí la importancia de continuar con acciones de lucha por parte de estos trabajadores, más aún cuando las condiciones laborales de estas empresas se mantienen para las nuevas generaciones, los hijos, los sobrinos y los nietos de estos. Dice el entrevistado:

- Como hablaba yo ahora por el megáfono, trajeron mulas, innovaron mulas para un trabajo bananero que se llama acarrear la fruta, y las mulas no dieron a vasto, porque no dieron la eficiencia del ser humano, entonces cambiaron las mulas, $y$ volvieron a traer a los trabajadores. Entonces eso quiere decir que 
nosotros somos altamente eficientes, más superiores que una mula, eso es lo que somos. Entonces venimos a ser como burros. Yo llamo a eso ser burros, y esos burros usted los va a ver en todas las fincas bananeras, que son los carreros, son hijos de nosotros, son nietos de nosotros, son sobrinos de nosotros, porque nosotros, los viejos trabajadores, ya no existimos para las bananeras, ya estamos viejos, ya nos discriminan, ya nos indican qué tipo de labor debemos hacer, ya no somos bien remunerados, somos maltratados. Y hoy día hay leyes de persecución laboral, de hostigamiento laboral, de esto de las mujeres, de la mano de obra infantil, pero no se dan cuenta que a nosotros los adultos bananeros nos están maltratando también (Hombre, trabajador afectado por el nemagón, 12/04/2012).

Este llamado a entender que las condiciones laborales deplorables para los trabajadores de la producción bananera persisten, llevó al Secretario General del Sindicato de Trabajadores de las Plantaciones (sitrap), ya fallecido, Carlos Arguedas, a denunciar las relaciones existentes entre esta producción y la producción piñera. Al respecto él señaló:

...Si el nemagón fue una tragedia que vivieron los trabajadores bananeros, yo les digo que las piñeras es la peor desgracia que nos hayan traído a la provincia de Limón y yo estoy profundamente convencido $y$ lo digo con conocimiento de doce años de estar lidiando, de doce o quince años de estar lidiando con esto, que definitivamente aquí en esta provincia de Limón debieran prohibirse totalmente la siembra de piña, debiera ser así... (Arguedas, Boeglin y Díaz, 2009).

\section{SITUACIÓN DE LA PRODUCCIÓN DE PIÑA EN LA REGIÓN CARIBE 2006-2012}

La producción de piña se ha expandido aceleradamente en el país en los últimos 20 años. Así, se estima que entre 1990 y el 2010, los territorios ocupados por el monocultivo de la piña aumentaron en un $700 \%$ (Fernández, 2012). En Costa Rica, las hectáreas dedicadas a esta siembra ya sobrepasan las 50 000, alcanzando ya el $1 \%$ del país. Este monocultivo se ha convertido en el de mayor expansión en los últimos años; en el 2007, se estiman que existían 29180 Ha y para el 2010, ya se registraban más de 45000 Ha (sEPSA, 2011 y Barquero, 2011). De acuerdo a cálculos de varias organizaciones ambientales, después de 2010, (y agregando varias hectáreas que no entran en los datos oficiales), las hectáreas dedicadas a este cultivo se acercan a las 60000 (Rojas, 2009).

En el Caribe costarricense, en los últimos años, la siembra de esta fruta ha aumentado considerablemente desencadenando otros problemas, como sucedió recientemente en La Curía de Roxana de Pococí, donde se han sembrado durante el 2012, al menos $400 \mathrm{Ha}$ sin permisos, debido a que existe una moratoria, transformando los 13 asentamientos campesinos $y$ destruyendo otras posibilidades de actividades económicas (Chavarría, 18/07/2012). Por este motivo es que se realiza esta valoración entre actores (comunidades - trabajadores y no trabajadores-, instituciones $y$ piñeras) involucrados en este proceso de expansión, a través de los conflictos surgidos entre ellos ${ }^{6}$.

La zona de Guácimo, Siquirres, Matina y comunidades aledañas se caracteriza por haber vivido en medio de plantaciones desde hace décadas, primero por las bananeras (que todavía continúan, en especial en el cantón de Matina ${ }^{7}$ ) $y$ recientemente, por el monocultivo de la piña,

6

Para ello se revisó la prensa, fundamentalmente, el Semanario Universidad, así como, los documentos y guías sobre esta temática, así como, investigaciones $y$ reflexiones de organizaciones ambientales y universidades públicas. La revisión de medios de prensa se realiza sobre los conflictos que han tenido lugar en el último quinquenio en la zona Caribe del país, principalmente en las poblaciones por las que pasa la línea del tren (o la carretera 32): Guácimo, Siquirres, Matina y comunidades aledañas.

Matina es el cantón productor de banano número uno, ya que las plantaciones de esta fruta superan las $10000 \mathrm{Ha}$. 
lo que ha desencadenado una serie de conflictos con los y las habitantes de las diferentes comunidades. Entre los principales problemas que argumentan los y las vecinas de las piñeras se encuentran la eliminación de las prácticas agrícolas tradicionales y por lo tanto, la pérdida de la soberanía alimentaria, la concentración de tierras en manos de grandes corporaciones, los problemas de salud debido a las constantes fumigaciones que se realizan sobre estas plantaciones, la contaminación de las afluentes de agua por los químicos utilizados en estos monocultivos, la tala de bosque y pérdida de árboles autóctonos, el aumento de sedimentación de los ríos, lagunas y humedales, producto de la erosión; el irrespeto de las garantías laborales y la evasión de las cargas sociales. La contaminación hace que se pierdan hectáreas de bosques, mientras los residuos de pesticidas afectan a los animales y los mantos acuíferos, en donde se han identificado más de 22 agro tóxicos peligrosos. Por último, se ha dado la aparición de nuevas plagas que afectan el ganando como la llamada mosca "chupasangre" (stomoxys calcitrans) (Picado, 2012).

Costa Rica es un país que dedica aproximadamente, el $10 \%$ del total de la superficie terrestre a la producción de cultivos agrícolas. Pero su agricultura, al ser extensa $y$ de pocos cultivos, es "químicamente muy intensa", reflejado sobre todo en las 9000 toneladas métricas anuales de plaguicidas que son importados al país (cica, Cimar, pNuma, minaet, 2009). En la provincia de Limón, esta actividad es muy importante, ya que de las 450000 personas que viven en la zona, el $30 \%$ viven $y$ trabajan en el sector agrícola, mientras que la pobreza se mantiene en el $29 \%$ y la tasa de desempleo en un 10\% (InEc, 2011). De las $45000 \mathrm{Ha}$ de piña registradas por el Ministerio de Agricultura y Ganadería, cerca de 15000 se encuentran en el Caribe, principalmente, en los cantones de Pococí, Guácimo, Siquirres y Matina.

Desde el 2003, se han realizado estudios en esta región que muestran altos índices de contaminación en sus fuentes de agua, asociado a la siembra de piña. Así:
... en el 2003 el Instituto Regional de Estudios de Sustancias Tóxicas de la Universidad Nacional (IRET) evidenció la presencia del toxico bromacil en las aguas superficiales. Pero es hasta julio del 2007 que se empieza a suministrar el agua en camiones cisterna. Esta contaminación del agua nos está costando según AyA, unos 15 millones al mes (350 millones en un año) para sufragar los gastos por dispensar agua en cisternas $y$ un deterioro aún desconocido en la salud de los habitantes (Kioscos Ambientales, 2009).

Por su parte, un estudio más reciente de la bióloga Silvia Echeverría afirma que lo más preocupante es "que aparecieron hasta siete plaguicidas diferentes en una sola muestra de agua, combinación que podría provocar un efecto tóxico mucho mayor del que producirían de forma independiente" (Blanco, 2011).

Ante los cuestionamientos que han realizado las comunidades y las organizaciones ambientales, las entidades estatales del sector agrícola y las piñeras han presentado una serie de documentos que intentan justificar el uso extensivo de las tierras o de promover un uso "racional" y "sostenible" de la tierra y los residuos de este cultivo. Un ejemplo es el compromiso público socio ambiental promovido por la Comisión Socio Ambiental de la Piña (cosap) y la Cámara Nacional de Productores y Exportadores de Piña (canapep), quienes se han comprometido a cumplir con las leyes y reglamentos, en especial, con la legislación ambiental y social vigente. Dentro de las medidas se ha anunciado conservar los recursos naturales: agua, aire $y$ suelo; promover la protección de la salud, higiene y seguridad de los colaboradores en las fincas; mitigar los efectos del Cambio Climático mediante la reducción de la huella de carbono en las empresas; proveer niveles de protección al ambiente, la inocuidad a la salud y seguridad para los colaboradores que participan en la producción y los miembros de las comunidades influenciadas por las operaciones productivas.

En términos de cambios productivos se ha propuesto: mejorar las regulaciones internacionales en protección de la salud 
y los estándares de inocuidad de los mercados internacionales; reducir los desechos sólidos y líquidos; utilizar prácticas de agricultura sostenible; utilizar sólo plaguicidas, productos orgánicos y biológicos autorizados por la legislación nacional e internacional; capacitar al personal relacionado con el proceso de producción, en cuanto al uso correcto, almacenamiento $y$ aplicación de las sustancias químicas, biológicas o afines para la prevención y combate de plagas, así como en la prevención de riesgos laborales; establecer el manejo del recurso hídrico para garantizar la calidad de las aguas residuales y superficiales del área de siembra; establecer técnicamente el manejo de suelos para reducir la erosión y sedimentación en el cultivo (canapep, 2012a: 9).

La Revista Piña de Costa Rica de canapep (en junio de 2012, cuentan con 10 ediciones) es otro medio que ha tratado de posicionar la labor de responsabilidad social y ambiental, mostrando cómo la economía depende de la piña en los cantones donde se cultiva. En el último número de esta revista, el editorial comienza con el siguiente párrafo:

Costa Rica ha sido un país de vocación agrícola, sin embargo, pocas actividades han sido tan cuestionadas por parte de la población civil y política, como la producción de piña. Se le ha cuestionado por el uso del suelo, por irrespeto a la legislación laboral y ambiental, por el uso de agroquímicos, como si solo esta actividad los utilizara, $y$ se obstaculiza la expansión del cultivo en parte del territorio (Chaves, 2012: 4).

En esta revista se evidencia cómo las empresas otorgan becas a niños y niñas, invierten en carreteras e infraestructura escolar. En este medio, las empresas destacan su compromiso socio ambiental y la importancia de conocer la huella ambiental del cultivo piñero ${ }^{8}$. Estos elementos componen parte de la estrategia de respuesta de CANAPEP a los cuestionamientos desarrollados dentro de la actividad piñera.

Por su parte, las instituciones públicas han sido incapaces de solventar los problemas $y$ las demandas de muchas comunidades que viven entre las piñeras. Como respuesta a esto, las instituciones han publicado una serie de documentos que justifican el uso de agroquímicos y varias prácticas integradas en un discurso de conciencia ambiental (BANACOL/ REPCAR). Ante las múltiples denuncias de las comunidades, el Ministerio de Agricultura y Ganadería (маG) implementó el "Manual de Buenas Prácticas Agrícolas para la Producción de Piña", un documento que no es vinculante y que se encarga de hacer una serie de recomendaciones como la siguiente:

Analizar al menos dos veces al año la calidad microbiológica y físico-química de las fuentes de agua que se están utilizando (pozo, canal abierto, embalses, ríos, lagos, etc.) y comprobar que se está cumpliendo con lo estipulado en la norma que para tal efecto establece el Ministerio de Salud. La frecuencia de los análisis variará en función del origen del agua $y$ de los riesgos de contaminación ambiental, incluida la contaminación temporal o intermitente (lluvias torrenciales, inundaciones, etc.). Además deben mantenerse registros de los resultados y de las medidas correctivas en el caso de no cumplir con las normas establecidas (маG, 2010: 59).

El cultivo de la piña representa el segundo cultivo de mayor producción en el país del sector agrícola, solo superada por la caña de azúcar, con 1976755 toneladas métricas. Según datos del mag, la piña representa el 15,3\% de la participación en el valor bruto del sector (SEPSA, 2011), cuyo "valor de las exportaciones de

$8 \quad$ Ver: Chaves, Abel. "La piña y crece y los pueblos se desarrollan o Empresas piñeras invierten en calidad estudiantil". Cámara Nacional de Productores

y Exportadores de Piña (CANaPeP). San José, Costa Rica. 2012: 3. 
la fruta se incrementó en un $55 \%$, pues pasó de $\$ 430$ millones, en el 2006, hasta $\$ 666$ millones en el 2010" (Barquero, 08/08/2011) y continúa creciendo, ya que para el 2011 se reportaron ganancias de $\$ 724$ millones y a mayo de 2012 , se han reportado $\$ 360$ millones (CANAPEP, $2012 b$ ). Las ganancias de estas actividades no se ven reflejadas en el desarrollo de estas comunidades, ya que de acuerdo a los datos del Índice de Desarrollo Humano Cantonal de Costa Rica, los cantones productores de piña del Caribe presentan la siguiente situación: Siquirres ocupa el puesto 62, Guácimo el 67, Pococí el 75 y Matina el 79 (PNUD, 2011).

A partir de la revisión realizada, se constata tal y como se plantea en el Informe del Estado de la Nación del año 2010: "en el ámbito agrícola, el cultivo de la piña ha sido el principal generador de denuncias formales de tipo ambiental". El Tribunal Ambiental Administrativo (TAA), por ejemplo, señala que en agosto de 2010, recibieron más de 400 denuncias por impacto ambiental de la producción de piña, específicamente, en los cantones de Limón: Siquirres, Matina, Guácimo y Pococí. Vale la pena aclarar que se trata de 400 denuncias recibidas, lo que no significa que todas sean tramitadas (TAA, 2010).

La principal denuncia que realizaron las comunidades estuvo vinculada a la contaminación del agua por residuos de plaguicidas utilizados por las empresas piñeras, con las fuertes repercusiones en la salud de estas personas. Como se mencionó, en la investigación realizada por el IRET-UNA, en sus análisis biológicos y físico-químicos, en varios puntos de la parte baja de la cuenca del Río Jiménez, entre los cantones de Pococí y Guácimo, se confirmó en ríos y quebradas, la presencia de residuos de plaguicidas utilizados en el cultivo de la piña (Blanco, 22/06/2011).

A principios de junio de 2010, personas vinculadas al acueducto rural de Milano, Siquirres, en compañía del Centro de Derecho Ambiental y Recursos Naturales (CEDARENA), demandaron a empresas como Del Monte y a la finca Babilonia por contaminación de aguas. El 29 de mayo de 2009, la Sala Constitucional ordenó sanear las aguas que estaban contaminadas de bromacil y otros 25 químicos más.
Asimismo, comunidades de El Cairo, La Francia, Siquirres, Luisiana y Milano, denunciaron la contaminación del agua de ríos y nacientes de esta zona, por falta de planta de tratamiento de aguas por parte de la Corporación de Desarrollo Agrícola Del Monte y los escasos e ineficientes controles ambientales que existen en el país. En esta ocasión se ordenaron medidas cautelares a la empresa $y$ orden de cierre de la planta empacadora de piña; sin embargo, algunas personas de estas comunidades aseguran que siguieron empacando producto (Córdoba, $25 / 05 / 2009$ y 02/06/2009). Estas poblaciones argumentan vivir problemas de salud y demandan un acueducto, para lo que no hubo una respuesta inmediata.

Por su parte, Acueductos y Alcantarillados (AyA) distribuye agua en cisternas, lo que resulta insuficiente para resolver las necesidades cotidianas de la población. Estas comunidades se manifestaron en la ruta 32 (Limón-San José) a mitad de 2009, exigiendo la pronta construcción del acueducto. En esta oportunidad, el gerente de AyA firmó el compromiso de iniciar la construcción de este, el $1^{\circ}$ de julio de 2009 , lo que no se ha cumplido por dificultades para la expropiación, compra y catastrado de terrenos, que pertenecen a empresas productoras de piña. Mientras tanto, estas comunidades tienen alrededor de tres años recibiendo el agua potable por medio de cisternas.

Otras comunidades como en Santa Rosa de Pococí, en enero de 2011, interpusieron un recurso de amparo ante la Sala iv, exigiendo un estudio de impacto ambiental e hidroeléctrico a cualquier empresa de producción de piña que pretenda instalarse en esta zona. Se declaró con lugar el recurso. La comunidad interpuso una acción legal que ha paralizado la siembra de piña en este lugar (Núñez, 18/01/2011).

La proliferación de la mosca hematófaga o mosca "paletera" es otra de las secuelas que generan las fincas piñeras, particularmente, las de Guácimo y Pococí. En este sentido, ganaderos de Río Jiménez, Villa Franca y San Luis (Guácimo), desde mediados del año 2009, denuncian $y$ demandan al mag $y$ el Servicio Nacional de Seguridad Animal (senasa), el cumplimiento de las normas de sanidad. Esta mosca 
causa la muerte de su ganado y no se conoce una respuesta al respecto. Ante una denuncia de un ganadero particular que denunció a Finca Sebastapol, senasa cerró la finca durante 3 días; de manera que las comunidades afectadas reclaman cumplimiento de normas impuestas para la reducción de moscas.

El hecho que las comunidades afectadas focalicen sus denuncias en la contaminación del agua $y$ en el caso de los ganaderos, la propagación de la mosca "paletera", no significa que sean los únicos daños que provocan la producción y expansión piñera. Como se mencionó, la invasión de áreas de protección y la aceleración del proceso de erosión de suelos y como consecuencia, la sedimentación de ríos, así como, el desplazamiento de cultivos de hortalizas y la afectación de la seguridad alimentaria, son también secuelas importantes del monocultivo de la piña.

Es importante mencionar que las comunidades afectadas por este monocultivo han recurrido a la figura de la moratoria para impedir la expansión piñera y así sus consecuencias. Esta petición de moratoria: "exige que las autoridades involucradas en el tema, como los ministerios de Salud, Agricultura y Ambiente, Energía y Telecomunicaciones, la SETENa $y$ las compañías piñeras, protejan la naturaleza y la salud de los habitantes", lo que en otras palabras significa el cese de la expansión piñera. Es así como el Concejo Municipal de Siquirres acordó esta moratoria el 17 de febrero de 2009; el Concejo Municipal de Pococí la acordó el 12 de marzo de 2012 y el Concejo Municipal de Guácimo rectificó la moratoria por dos años más, el 27 de abril de 2012; es decir, el acuerdo de moratoria se dio en abril de 2010 (Ramírez, 04/03/2009).

Existen otros actores en el escenario del monocultivo de la piña que deben mencionarse; por ejemplo, el señor Abel Chaves, Presidente de la Cámara Nacional de Productores y Exportadores de Piña (canapep), quien ante las denuncias presentadas por comunidades y grupos ecologistas, manifestó que:

... la producción de piña ha contribuido al desarrollo del país, da empleo a al menos 137500 personas, puso en práctica el Sistema de Gestión Socio ambiental
(2008), convenios para impulsar la producción sostenible con: el INBIO, TEC, TAA, Mag $y$ con la Cooperativa de Profesionales del Colegio de Ingenieros Agrónomos. Se rige por normas nacionales e internacionales que rigen la producción (07/12/2011).

Por su parte, el Consejo Universitario de la Universidad de Costa Rica, en diciembre de 2008, emitió un pronunciamiento en el que solicitó una moratoria inmediata para nuevas plantaciones de piña en todo el territorio nacional (acuerdo de la sesión cu-5318). canapep ha señalado que la Universidad no debería propiciar actividades como las que realiza Kioscos Socioambientales (otro actor muy importante en el acompañamiento a las comunidades denunciantes) $y$ alegan que las piñeras brindan trabajo a 27000 personas de forma directa y a 110000 indirectamente, aduciendo prácticas sostenibles (Córdoba, 24/03/2009).

Finalmente, el Frente Nacional de Sectores Afectados por la Producción Piñera (FRENASAPP), ganaderos y personas de las comunidades del Caribe y la Zona Norte afectados por las malas prácticas en la producción de piña, en octubre de 2009, denunciaron ante la Misión Dirección General de Inocuidad de Alimentos y Protección al Consumidor (DG-SANCo) de la Unión Europea (UE), los efectos del cultivo de la piña en el ambiente y el agua. Se basan en resultados de los análisis del IRET, denuncias penales de organizaciones contra fincas piñeras, fotografías de los incumplimientos, la deforestación y testimonios de las comunidades afectadas. Aún no se conoce respuesta a esta denuncia (Córdoba, 09/10/2009).

\section{REFLEXIONES FINALES}

En este artículo se buscó posicionar un recuento en torno a las recientes conflictividades que han surgido en el Caribe costarricense, a través de tres perspectivas distintas: las luchas por la tenencia de la tierra, el monocultivo del banano y el monocultivo de la piña. Sin pretender agotar la discusión, se presentan algunas ideas para la reflexión, a 
fin de posicionar y repensar la situación actual del Caribe costarricense.

La primera de las ideas refiere a la continuidad y permanencia de los conflictos del Caribe. Como da cuenta la síntesis presentada en este trabajo, las disputas surgidas en torno a los monocultivos y por la tenencia de la tierra en el Caribe, no son disputas nuevas en la historia de las conflictividades de la región. Por el contrario, muchos de los conflictos se mantienen sin resolver, lo cual es particularmente cierto en el tema de las disputas por la tierra $y$ en el monocultivo del banano. No es esta la situación con la producción de la piña, fenómeno relativamente reciente en la historia de Costa Rica; sin embargo, conserva muchas características de la producción de monocultivos, en general.

Es así que las propuestas de proyectos de ley o el surgimiento de nuevas normativas, la inserción y el proceder de la institucionalidad pública, la relación del Estado con grupos de la región (campesinos, indígenas o trabajadoras y trabajadores bananeros o piñeros y comunidades, en general) y el desarrollo de capacidades organizativas alrededor de los distintos ejes que estructuran el presente estudio, con su correspondiente persecución y represión, así como, el desarrollo de determinadas enfermedades o efectos en la salud de las actividades productivas vinculadas a los monocultivos, son procesos que han atravesado la historia de la región y que se mantienen vigentes.

Lo anterior no niega las especificidades que tiene cada uno de los ejes de la conflictividad expuestos, así como la concreción geográfica de estos conflictos en zonas diferenciadas del Caribe Norte y el Caribe Sur, que sería una segunda idea para la discusión. En este sentido, los conflictos en torno a la producción de piña se encuentran ubicados en el Caribe Norte, tanto como la producción bananera. Por su parte, la existencia de territorios indígenas $y$ zonas protegidas en el Caribe Sur ha hecho que las formas de resistencia indígena se ubiquen en este espacio. De la misma manera, las categorías de protección de gran parte de esta zona han permitido mitigar las posibilidades de avance de los monocultivos. Paradójicamente, el mismo sistema de mitigación ha hecho que exista poco espacio disponible para el desarrollo de actividades económicas, sociales y productivas locales, por lo que la presión por el uso del espacio y sus recursos es mayor. De esta manera cualquier cambio en la administración del territorio, como es el caso de la modificación de la legislación de los asentamientos campesinos, puede tener un impacto muy sensible en la vida cotidiana de sus pobladores que dependen en su mayoría de la tierra.

Una tercera idea para la discusión, se relaciona con la necesidad de profundizar en las formas de intervención o no intervención estatal en el Caribe. Se considera que este es un aspecto que merece una mayor indagación, siendo fundamental entender cómo la región Caribe ha recibido una atención diferenciada, en cuanto a los conflictos surgidos en esta zona del país. De la misma manera, estas discusiones permitirían reflexionar sobre el lugar que la región caribeña ha ocupado en el país, en términos económicos, sociopolíticos y también imaginarios.

En cuarto lugar, a partir del análisis regional del Caribe en torno a los conflictos analizados, es central considerar el tema del Estado $y$ los costos sociales, económicos y subjetivos que tiene de la producción bananera y piñera. ¿Quién asume los costos sociales de la producción bananera y piñera?, ¿cuál es el papel de la institucionalidad pública, en este caso del Instituto Costarricense de Acueductos y Alcantarillados (AyA), el Ministerio de Salud, la Caja Costarricense de Seguro Social (ccss) y de las municipalidades en dichos procesos?, ¿cuánto le cuesta al Estado la producción bananera y la producción piñera? y ¿con qué recursos cuentan las comunidades y las familias para enfrentar la contaminación, el acceso restringido al agua $y$ las afectaciones que estas producciones dejan en la salud? Estas son algunas de las preguntas que interesan en este cuarto aspecto $y$ que requieren de una mayor indagación con perspectiva regional.

Finalmente, un último aspecto relacionado directamente con el anterior, refiere a las diferencias en las formas y estrategias con las que se lucha, en las áreas analizadas en este 
trabajo. En el tema de la lucha contra la expansión piñera, ha tenido una gran importancia la figura de la moratoria a la producción de piña. La moratoria ha traído a las comunidades que luchan contra la expansión piñera, la esperanza $y$ la posibilidad de ver resultados concretos, producto de sus estrategias de lucha y sus acciones de resistencia. Este recurso de carácter legal no está disponible en la lucha por la tenencia de la tierra y contra la expansión bananera y sus consecuencias. En el caso de las luchas contra el monocultivo del banano y sus efectos, se señala la dificultad de encontrar en el recuento reciente de las luchas en este ámbito, acciones que de manera significativa cuestionaran la producción bananera, las condiciones laborales $y$ los efectos en la salud. Se señalaba también que este proceso puede estar relacionado con la ausencia de fuentes de empleo en la región, las amenazas de pérdida de empleo ante la posibilidad de organización sindical o de otro tipo, y una "naturalización" y "aceptación" de que las condiciones en las cuales se produce el banano son desiguales e injustas. Esto lleva a replantear también, qué tipo de organización por parte de los trabajadores de la piña, está surgiendo en la región.

\section{BIBLIOGRAFÍA}

Amador, Marvin. "Banano que envenena. Triste record para Limón". Revista Foro. Revista de Ciencias Sociales y Jurídicas. Madrid, España. Universidad Complutense de Madrid, 1998: 23-25.

Asamblea Legislativa. Proyecto de Ley nro. 17 956: Concesión de la zona fronteriza con Panamá. San José, Costa Rica: Departamento de Servicios Parlamentarios, 2010.

Arguedas, Carlos; Boeglin, Nicolás y Díaz, Hazel. "Del nemagón al bromacil: monocultivos, luchas sociales $y$ ambiente". Ponencia. [Audio]. Mesa Redonda Instituto de Investigaciones Sociales (IIS). San José, Costa Rica. 22 de setiembre de 2009.

banacol-repcar. "Manual de Buenas Prácticas Agrícolas para la Producción de Piña en Costa Rica”. Proyecto Colombia,
Costa Rica, Nicaragua: reduciendo el escurrimiento de plaguicidas al mar Caribe. Banacol, s.f. En: <http://cep.unep. org/repcar/proyectos-demostrativos/ costa-rica-1/publicaciones-banacol/ Manual\% 20 в РА $\% 20$ Banacol.pdf $>$ [consultado el 4 de julio de 2012].

Barquero, Marvin. "Área sembrada de piña se duplicó en últimos cuatro años". La Nación. San José, Costa Rica. 08 de agosto de 2011.

Barquero, Marvin. "Trabajadores paralizaron tres fincas bananeras de Del Monte". La Nación. San José, Costa Rica. 10 de noviembre de 2011.

Barquero, Marvin. "Acuerdo de Bandeco y sindicato pone fin a huelga en Sixaola". La Nación. San José, Costa Rica. 01 de diciembre de 2011.

Barraza, D., Jansen, K.; van Wendel de Joode, B. y Wesseling, C. "Social movements and risk perception: unions, churches, pesticides and bananas in Costa Rica. International journal of occupational and environmental health" Impact Factor 19(1). 2013: 11-21. En: <http://www.researchgate. net/publication/236198967_Social_ movements_and_risk_perception_ unions_churches_pesticides_and_ bananas_in_Costa_Rica>.

Blanco, Patricia. "Detectan plaguicidas en agua de zona piñera en Limón". Semanario Universidad. San José, Costa Rica. 22 de junio de 2011.

Boeglin, Nicolás. "Nivel de cumplimiento de decisiones judiciales en materia ambiental relativas a la protección del recurso hídrico". Actas de ponencia del Decimosexto Informe Estado de la Nación. San José, Costa Rica: Programa Estado de la Nación, 2010.

Bogantes, Javier. "Perspectivas para un banano justo". La voz del manatí. Boletín informativo del Foro Emaús 11. Costa Rica, 1997: 6-7.

Borge, Carlos y Villalobos, Victoria. Talamanca en la encrucijada. San José, Costa Rica: Editorial de la Uned (EUNED), 1998. 
Bourgois, Philippe. Banano, etnia y lucha social en Centro América. San José, Costa Rica: Departamento Ecuménico de Investigaciones (DEI), 1994.

Cámara Nacional de Productores y Exportadores de Piña (canaper). Exportaciones de piña fresca. San José, Costa Rica: canaper, 2012a.

Cámara Nacional de Productores y Exportadores de Piña (canapep). Sistema de gestión socio ambiental para la producción sostenible de la piña. San José, Costa Rica: canapep, 2012b.

Centro de Investigación en Contaminación Ambiental (cica), Centro de Investigación en Ciencias del Mar y Limnología (сімAR), Ministerio de Ambiente y Energía (мINAET) y Programa de las Naciones Unidas para el Medio Ambiente (PNUma). "Reducción del escurrimiento de plaguicidas al mar Caribe". Informe Técnico. San José, Costa Rica. 2009.

Céspedes, Walter. Proyecto de Ley nro. 18 425: Declaratoria de Cahuita y Puerto Viejo como ciudad. San José, Costa Rica: Departamento de Servicios Parlamentarios, 2012.

Céspedes, Walter. Proyecto de ley nro. 18 207: Reconocimiento de los derechos de los habitantes del Caribe. San José, Costa Rica: Departamento de Servicios Parlamentarios, 2011.

Chacón, Vinicio. "Campesinos de Sixaola temen que el IDA les quite sus tierras". Semanario Universidad. San José, Costa Rica. 13 de julio de 2011.

Chacón, Vinicio. "Indígenas de Kekoldi en Talamanca reclaman su territorio". Semanario Universidad. San José, Costa Rica. 10 de agosto de 2011.

Chavarría, David. "Denuncian expansión agresiva de monocultivo de piña en el Caribe". Semanario Universidad. San José, Costa Rica. 18 de julio de 2012.

Chaves, Abel. "Responsabilidad social y ambiental". Piña de Costa Rica. El dulce orgullo de los ticos 10. San José, Costa Rica. Cámara Nacional de Productores y Exportadores de Piña (CANAPEP), 2012: 4.
Chaves, Abel. "Progresamos con desarrollo social y mejoramiento ambiental". Semanario Universidad. San José, Costa Rica. 07 de diciembre de 2011.

Comité de Seguimiento del Conflicto Social y la Coyuntura Latinoamericana de Costa Rica (osal). "Cronología del conflicto social". Documento de trabajo nro. 538. Consejo Latinoamericano de Ciencias Sociales (clacso), setiembre 2009.

Consejo Nacional Trabajadores. "Contaminación de aguas por el Nemagón. Aprendamos de la experiencia de otros". La voz del manatí. Boletín informativo del Foro Emaús 17. Costa Rica, 1999: 4.

Coordinadora de Sindicatos Bananeros. "Represión en la zona atlántica costarricense". Revista Foro. Revista de Ciencias Sociales y Jurídicas. Madrid, España. Universidad Complutense de Madrid, 1998: 14-18.

Cordero, Allen. Los movimientos campesinos costarricenses vistos a través de tres casos de asentamientos del IDA. San José, Costa Rica: Facultad Latinoamericana de Ciencias Sociales (flacso), 2011.

Córdoba, Javier. "Piñeros responden a solicitud de moratoria de la ucR". Semanario Universidad. San José, Costa Rica. 24 de marzo de 2009.

Córdoba, Javier. "Indígenas denuncian amenazas de desalojo en territorio de Keköldi”. Semanario Universidad. San José, Costa Rica. 14 de abril de 2011.

Córdoba, Javier. "Laboratorios de la una comprobaron contaminación de piñera" Semanario Universidad. San José, Costa Rica. 26 de mayo de 2009.

Córdoba, Javier. "Comunidad exige derecho a tomar agua potable". Semanario Universidad. San José, Costa Rica. 2 de junio de 2009.

Córdoba, Javier. "Comunidades y organizaciones: piden a europeos ampliar investigación sobre la piña”. Semanario Universidad. 20 de octubre de 2009.

Coto, Alfonso. "Alto a la expansión bananera incontrolada. Carta Pastoral de Mons. 
Alfonso Coto Monge, obispo de Vicario Apostólico de Limón y presbíteros. 25 de diciembre de 1989, en la fiesta de la Navidad". Foro Emaús. 10 años de lucha. Siquirres, Costa Rica. Foro Emaús, 2003: 23-33.

Díaz, Luis. "INs deberá examinar a afectados por el Nemagón”. La Nación. San José, Costa Rica. 22 de setiembre de 2010.

Fernández, Eduardo. "Un parque nacional de piña”. Semanario Universidad. San José, Costa Rica. 23 de mayo de 2012.

Fernández, Mario. "La estructura agraria de la región fronteriza de Costa Rica con Panamá: resultado de la lucha campesina por la tierra". Revista de Ciencias Sociales 45-46. San José, Costa Rica. Universidad de Costa Rica, 1989: 57-71.

Giralt, Marielos. "La problemática ética del uso del рвср en Costa Rica". Revista de Filosofía. San José, Costa Rica. Universidad de Costa Rica, 1996: 83-84.

Hermosilla, Hernán. "Historia del Foro Emaús. Un camino popular y ecuménico en defensa de la vida". Revista Foro. Revista de Ciencias Sociales y Jurídicas. Madrid, España. Universidad Complutense de Madrid, 1998: 8-13.

Instituto Nacional de Estadísticas y Censos (INEc). Región H. Atlántica año 2011. San José: Inec, 2011.

Kioscos Ambientales. "Criminalización en Costa Rica: ambientalista será enjuiciado". Kioskos Ambientales. San José, Costa Rica. Universidad de Costa Rica, 2009. En: <http:// kioscosambientales.ucr.ac.cr/index. php?option $=$ com_content $\& v i e w=$ article \&id=427:criminalizacion-en-costa-ricaambientalista-sera-enjuiciado\&catid= 42:ambientales\&Itemid $=57>$ [consultado el 7 de julio de 2012].

Llaguno, José y Picado, Henry. "Legalizar el despojo". Biodiversidad: sustento y culturas 69. Barcelona, España. grain, 2011: 17-18.

Marquardt, Steve. "Green havoc: Panama disease, environmental change, and labor process in the Central America banana industry". The American Historical Review 1 (106). Indiana, eeuu: American Historical Association, 2001.

Marquardt, Steve. "Pesticidas, pericos y sindicatos en la industria bananera costarricense. 1938-1962". Revista de Historia 47. Heredia, Costa Rica. Escuela de Historia de la Universidad Nacional (unA)-Centro de Investigaciones Históricas de América Central (сінас) de la Universidad de Costa Rica (UCR), 2003: 43-95.

Ministerio de Agricultura y Ganadería (maG). Manual de Buenas Prácticas Agrícolas para la Producción de Piña. San José, Costa Rica: мAG, 2010.

Ministerio de Agricultura y Ganadería (MAG). Planificación Estratégica Programa Direcciones Regionales.Región Huetar Atlántica 2003-2006. San José, Costa Rica: MAG, 2007.

Montero, Andrea y Viales, Ronny. "Una aproximación al impacto ambiental del cultivo del banano en el Atlántico/Caribe de Costa Rica (1870-1930)". Costa Rica: cuatro ensayos de historia ambiental. Ronny Viales y Anthony Goebel (eds.). San José, Costa Rica: Sociedad Editora Alquimia 2000, 2011.

Mora-Alfaro, Jorge. El Instituto de Desarrollo Agrario (IDA), el desarrollo productivo y bienestar social en el medio rural de Costa Rica. Heredia, Costa Rica: Programa Regional de Maestría en Desarrollo Rural, 2006.

Mora, Sindy. "Reflexiones para el análisis comparativo de movimientos sociales: el caso de las ex trabajadoras y ex trabajadores bananeros afectados por el nemagón en Costa Rica y Nicaragua". Anuario de Estudios Centroamericanos 39. San José, Costa Rica. Universidad de Costa Rica, 2013.

Municipalidad de Talamanca. Plan local de desarrollo de Talamanca 2003-2013. 2003.

Núñez, María José. "Comunidades buscan moratoria a siembra de piña". Semanario Universidad. San José, Costa Rica. 18 de enero de 2011. 
Picado, Henry. "Otro ejemplo de economía verde: la falacia de la piña "responsable"". Amigos de la tierra Costa Rica. Asociación Comunidades Ecologistas la Ceiba (соесосеiвa). Junio, 2012. En: $<$ http://coecoceiba.org/otro-ejemplo-dela-economia-verde-la-falacia-de-la-pinaresponsable/> [consultado el 13 de junio de 2012].

Programa de las Naciones Unidas para el Desarrollo (pnud). Atlas del Desarrollo Humano Cantonal de Costa Rica 2011. San José, Costa Rica: pnud Costa Rica, 2011.

Quesada, Erlinda. "La lucha de la mujer limonense. El silencio se vuelve gemido". Revista Foro. Revista de Ciencias Sociales y Jurídicas. Madrid, España. Universidad Complutense de Madrid, 1998: 37-39.

Ramírez, Ernesto. "Ambientalistas piden detener depredación en refugio GandocaManzanillo". Semanario Universidad. San José, Costa Rica. 23 de agosto de 2011.

Ramírez, Lucía. "Siquirres establece moratoria a actividad piñera". Semanario Universidad. San José, Costa Rica. 04 de marzo de 2009.

Rodríguez, Silvia. El despojo de la riqueza biológica: de patrimonio de la humanidad a recurso bajo la soberanía del Estado. México DF: Editorial Ítaca, 2012.

Rodríguez, Tania. "Actores en cuencas transfronterizas: dinámicas de cooperación y de conflicto en la gestion de cuencas transfornterizas en centroamérica". Ponencia presentada en Jornadas de Jóvenes Americanista. Territorios y sus desafios en América: perspectivas transdisciplinarias $y$ diálogos metodológicos. San José, Costa
Rica: Universidad de Costa Rica. 15 de junio de 2012.

Rojas, Isaac. "Los desastres de la piña en Costa Rica". Biodiversidad, sustento y culturas 61. Barcelona, España. GRAIN, 2009: 18-19.

Ruiz, Gerardo. "Limón ciudad-Puerto". El Financiero. San José, Costa Rica. 06 de septiembre de 2013.

Secretaria Ejecutiva de Planificación Sectorial Agropecuaria (sEPsa). Boletín Estadístico Agropecuario 21. Serie cronológica 20072010. San José, Costa Rica: Secretaria Ejecutiva de Planificación Sectorial Agropecuaria, 2011.

Tribunal Ambiental Administrativo de Costa Rica. Más de 400 denuncias ha recibido el Tribunal Ambiental según corte a agosto de 2010. 2010. En: <http://www. tribunalambiental.org/noticias/noticiasdel-2010/29-mas-de-400-denuncias-harecibido-el-tribunal-ambiental-seguncorte-a-agosto-de-2010> [consultado el 23 de julio de 2012].

Vargas, Gerardo. "La problemática socio ambiental bananera". Revista Foro. Revista de Ciencias Sociales y Jurídicas. Madrid, España. Universidad Complutense de Madrid, 1998: 4-7.

Villegas, Jairo. "Dole pagará indemnización a 780 afectados por Nemagón". La Nación. San José, Costa Rica. 12 agosto de 2011.

\section{ENTREVISTAS}

Hombre integrante y fundador del Foro Emaús. Realizada el 23 de enero de 2012.

Hombre trabajador afectado por el nemagón. Realizada el 12 de abril de 2012.

Mujer integrante fundadora del Foro Emaús. Realizada el 11 de julio de 2012.

Fecha de ingreso: 13/01/2014 Fecha de aprobación: 05/04/2014 